Proc. of the 15th Int. Workshop on Slow Positron Beam Techniques and Applications, Prague, September 2-6, 2019

\title{
Estimation of Ortho-Positronium Pick-Off Contributions to the Coincident Measurement of the Energy Spectra of Positron-Induced Electrons and Annihilation Gamma Quanta
}

\author{
R.W. Gladen ${ }^{a *}$, V.A. Chirayath ${ }^{a}$, A.J. Fairchild ${ }^{a}$, S. Lotfimarangloo ${ }^{a}$, T. $\operatorname{Shead}^{a}$,

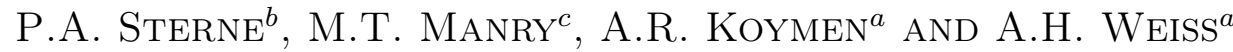 \\ ${ }^{a}$ Department of Physics, The University of Texas at Arlington, Arlington, TX, 76114, USA \\ ${ }^{b}$ Lawrence Livermore National Laboratory, Livermore, CA, 94550, USA \\ ${ }^{c}$ Department of Electrical Engineering, The University of Texas at Arlington, Arlington, TX, 76114, USA
}

\begin{abstract}
We present preliminary results that were collected using a new positron beam system at the University of Texas at Arlington. The beam allows for the coincident measurement of the energy of positron annihilation gamma photon and time-coincident positron-induced electrons. This system enables the parallel collection of a 2dimensional array of coincident pairs of gamma and electron energies over the full energy range of relevance. Here, we present gamma spectra constructed from the correlation of secondary electrons with the delayed annihilation of positronium, and the observation of pick-off annihilations that results from this analysis. These measurements will play an important role in determining the contributions due to pick-off annihilations to the Doppler-broadened gamma spectra from the internal or external exposed surfaces of materials. Subtracting the background properly is crucial for the measurement of the Doppler broadened spectrum of annihilation gamma photons originating from the annihilation of positrons with electrons of specific electronic core levels.
\end{abstract}

DOI: 10.12693/APhysPolA.137.101

PACS/topics: 78.70.Bj, 07.05.Kf, 79.20.Fv, 79.20.Hx, 81.05.ue, 82.80.Pv, 41.75.Fr, 71.60.+z

\section{Introduction}

Ortho-positronium (o-Ps) pick-off annihilations can occur when long-lived o-Ps encounters another surface and results when the positron within an o-Ps atom annihilates with an electron of the opposite spin through a variety of processes [1]. In the present apparatus [2], it is possible for a positron incident on a sample to induce secondary electron emission through impact ionization. This positron may then diffuse to the surface and pick up another electron before being re-emitted as o-Ps [3]; the gamma photon resulting from the annihilation of the re-emitted o-Ps may then be detected in coincidence with the detection of the positron impact-induced secondary electron. In the normal scenario (i.e. the gamma photon is a result of the annihilation of a positron with a bound electron in the material), the time difference between the detection of the annihilation gamma photon and the detection of the secondary electron is then taken as the time-of-flight of the electron and is representative of the electron energy. Although, there is an additional effect due to the relatively long lifetime of o-Ps: the decay time may be significant with respect to the flight time of the secondary electrons. This effect can thus result in a modification of the electron time-of-flight

*corresponding author; e-mail: randall.gladen@uta.edu
(ToF) spectrum in the form of an exponentially decaying tail [4]. Additionally, the longer-lived o-Ps atoms have some probability of reaching a surface within the sample chamber. These long-lived o-Ps atoms can then annihilate via pick-off, resulting in a background contribution to the gamma spectrum with an annihilation peak line shape that is independent of the sample material.

\section{Experimental apparatus}

The apparatus used to collect the data presented within this proceeding consists of a variable energy positron beam, a sample chamber, an electron time-offlight energy analyzer with an electron flight path of $\approx 3 \mathrm{~m}$, and a high-purity Ge detector (HPGe) (Fig. 1) [2]. Low energy positrons $(\approx 5 \mathrm{eV})$ are obtained from the moderation of fast positrons produced by the beta decay of a ${ }^{22} \mathrm{Na}$ source $[5]$ and are guided to the sample consisting of bilayer graphene on a substrate of polycrystalline $\mathrm{Cu}$ biased to $-50 \mathrm{~V}$ - with an axial magnetic field of $\approx 0.005 \mathrm{~T}$. The resulting positron-induced electrons are then guided to the micro-channel plate electron detector via a field-free region colloquially known as a ToF tube; the corresponding annihilation gamma ray is collected by the HPGe detector and the resulting detector pulse is used for both timing and energy measurements. Timing with the HPGe detector was enabled by an implementation of the extrapolated leading edge timing algorithm in the analysis software (a detailed description of which is provided in Ref. [6]), resulting in a timing 


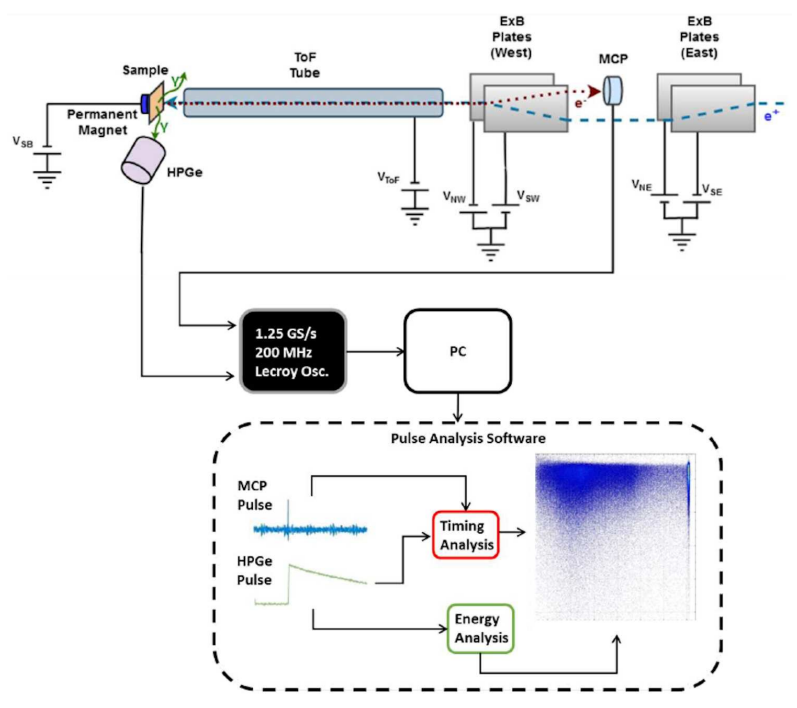

Fig. 1. Diagram illustrating the electron time-of-flight spectrometer and the flow of the detector signals. The ExB plates are used to drift the positrons beneath the MCP and back onto the axis of the beamline, as well as to drift the resulting positron-induced electrons into the MCP. The "ToF tube" serves as a field-free region, within which the electrons spend the majority of their flight time and travel without external influence from stray electric fields.

resolution of $16.3 \mathrm{~ns}$ for the HPGe and microchannel plate combination. The gamma energy of each individual positron-electron annihilation is thus correlated with the apparent time-of-flight $(\Delta t)$ of the positron impactinduced secondary electron. We measure this $\Delta t$ as the time difference between the detection of an HPGe detector pulse and a micro-channel plate (MCP) electron detector pulse via custom software that additionally constructs a two-dimensional array representing the coincident measurement of the electron ToF and the annihilation gamma energy [6].

\section{Data analysis}

Figure 2a is an electron ToF spectrum resulting from the application of the aforementioned settings. The longer decay time of o-Ps $(\approx 142 \mathrm{~ns})$, with respect to p-Ps $(\approx 0.125 \mathrm{~ns})$, will be reflected in the ToF spectrum as an exponentially decaying tail that extends beyond the flight times of secondary electrons ejected with the maximum possible energy $(\approx 105 \mathrm{eV})$. This effect can be used to produce the gamma spectrum due entirely to o-Ps formation by taking only the gamma counts that are associated with an electron arrival time of less than $\approx 560$ ns (corresponding to an electron energy of $\approx 105 \mathrm{eV}$ ). By selecting time ranges in the secondary electron spectrum (Fig. 2a) - and integrating the counts in the two-dimensional gamma-electron coincidence array along these regions [2] - multiple gamma spectra will result representing a variety of annihilation processes (Fig. 3). These integrations,
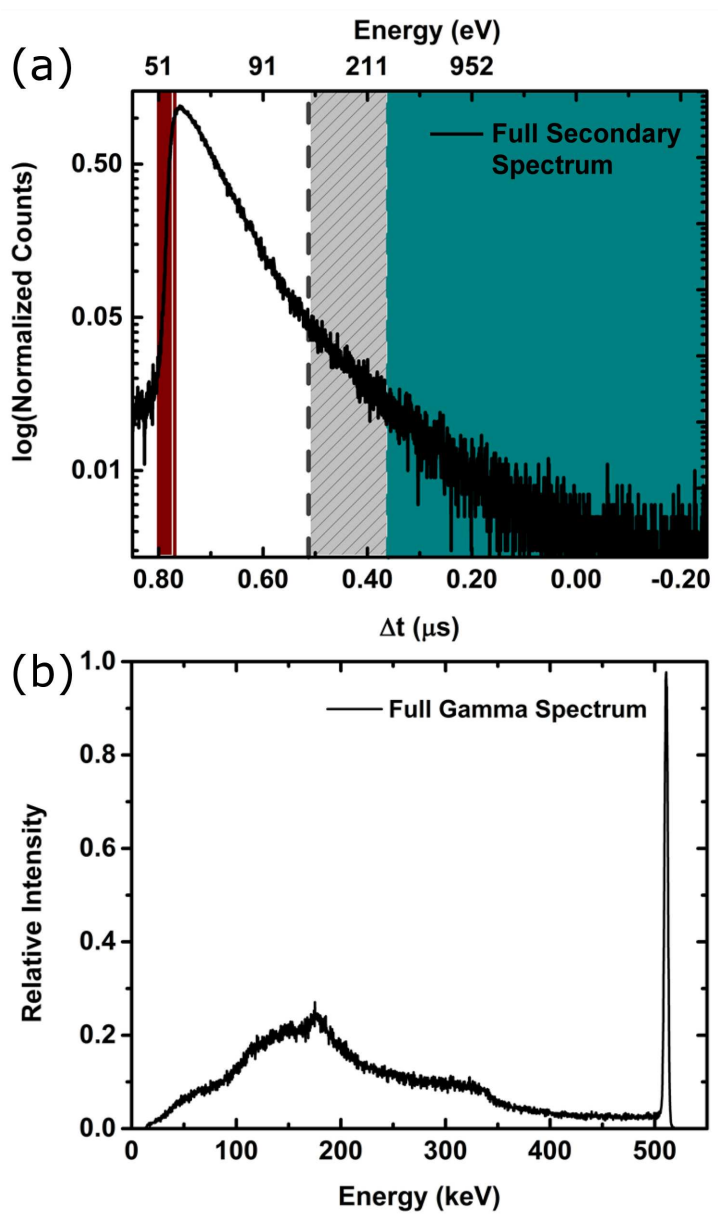

Fig. 2. (a) Secondary electron ToF spectrum for a $50 \mathrm{~V}$ sample bias illustrating the regions along which the cuts are made: "prompt" annihilations at 769-800 ns (red); "delayed" annihilations at 360-513 ns (grey hatch); and "extra delayed" annihilations at (-250)-350 ns (dark cyan). The "extra delayed" region represents the time at which the onset of pick-off annihilations appears to occur. The vertical axis is in natural log scale and the horizontal axis is the reverse time-of-flight of the electrons. (b) The full gamma energy spectrum collected in true coincidence with the secondary electrons.

or "cuts", were made along regions in the secondary spectrum of Fig. 2a that represent the "prompt" (red) and "delayed" (grey) annihilations of the positrons. Figure 3b is the full gamma spectrum collected in coincidence with the secondary electron ToF spectrum and is presented for comparison.

\section{Results}

\subsection{Prompt annihilations}

The prompt region of Fig. 2a corresponds primarily to either the annihilation of a positron with a bound electron in the sample or to the annihilation of p-Ps in addition to some contribution due to o-Ps annihilations. As p-Ps annihilation overwhelmingly results in $2-\gamma$ 

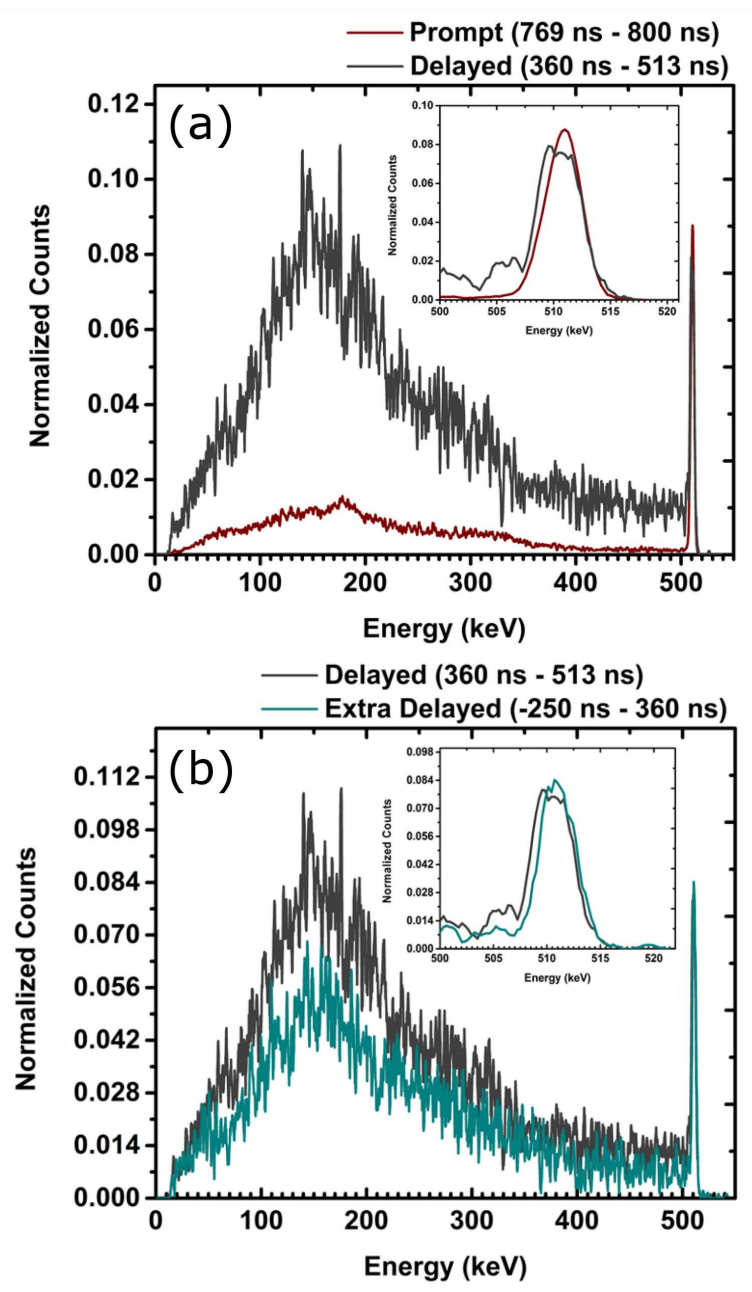

Fig. 3. (a) HPGe gamma spectra of prompt and delayed regions normalized to the intensity in the region between $509 \mathrm{keV}$ and $513 \mathrm{keV}$. (b) HPGe gamma spectra of delayed and extra delayed regions, illustrating the process of o-Ps pick-off on a surface within the sample chamber.

emission, collecting the gamma photons corresponding to this prompt region should result in a gamma spectrum with an enhanced $511 \mathrm{keV}$ annihilation peak and reduced intensity in the low energy region (corresponding to a reduction in o-Ps contributions). The red spectrum in Fig. 3a illustrates this case with a peak-to-total ratio defined here as the ratio of the integrated intensity between $511 \mathrm{keV}$ and $517 \mathrm{keV}$ and the integrated intensity of the entire spectrum - of $\approx 5.66 \%$. In comparison with the full gamma spectrum provided in Fig. 3b, which provides a peak-to-total ratio of $\approx 3.99 \%$, the cut along the prompt region results in an increase of $\approx 42 \%$ in the relative peak-to-total ratios.

\subsection{Delayed annihilations}

In contrast to the prompt annihilations, a cut along the delayed region of the secondary spectrum, which corresponds to the longer lifetime of o-Ps, should result in a gamma spectrum that exhibits a suppression of the $511 \mathrm{keV}$ annihilation peak and a corresponding enhancement of the low energy region intensity. This effect is due to the continuous energy distribution of o-Ps annihilation [7] and can be seen in the grey spectrum of Fig. 3a and $\mathrm{b}$ - the analysis of which yields a peak-to-total ratio of $\approx 0.89 \%$. Additionally, by cutting along the "extra delayed" region of the secondary spectrum (cyan box in Fig. 2a), the pick-off annihilations can be observed as a slight increase in the intensity of the $511 \mathrm{keV}$ annihilation peak, as shown as the cyan spectrum in Fig. 3b. The peak-to-total ratio of this "extra delayed" spectrum increases slightly to $\approx 1.55 \%$, indicating an increase in $2-\gamma$ annihilations. This enhancement in the $511 \mathrm{keV}$ peak can be attributed to an increase in the number of o-Ps atoms reaching a surface within the apparatus and annihilating via pick-off.

\section{Discussion}

A similar technique can be applied to ToF spectra resulting from the correlation of annihilation gamma photons with positron annihilation-induced Auger electrons ("gamma-Auger coincidence"). This application produces Doppler-broadened annihilation peaks corresponding to individual electronic core levels [2], but (due to the energy of the incident positron beam of $\approx 5 \mathrm{eV}$ ) low-energy secondary electrons that are correlated with o-Ps emission may also be produced and the pick-off annihilations of these o-Ps atoms will modify the shape of the resulting annihilation peak. We can see this effect in Fig. 2a: the Ps tail of the secondary electron spectrum extends beyond the maximum energy of the secondary electrons and even into "negative" time; and, as shown in Fig. 3b, longer Ps lifetimes correspond to an increase in the probability of pick-off annihilations. This contribution must therefore be measured and subtracted from the Dopplerbroadened annihilation spectra that result from correlating the annihilation gamma with individual core level annihilations.

\section{Conclusions}

The results of the peak-to-total measurements for the prompt region $(5.66 \%$ for the prompt spectrum and $3.99 \%$ for the original spectrum) indicate that the gamma spectrum due to prompt annihilations is primarily the result of $2-\gamma$ emission. Taking the spectra in Fig. 3a, the contribution of pick-off annihilations may be estimated by normalizing the $511 \mathrm{keV}$ annihilation peaks of the prompt spectrum and the sum (not shown) of the delayed and extra delayed spectra of Fig. 3b. The resulting ratio of the total integrated intensity of the prompt spectrum and the sum of the delayed and extra delayed spectra is $\approx 20.4 \%$. Pick-off annihilations should therefore comprise $\approx 20.4 \%$ of the Ps tail beyond the maximum energy of the electrons in the secondary electron spectrum. It should be noted again that this result is specific for 
a sample of bilayer graphene on $\mathrm{Cu}$ in our particular apparatus. These results suggest that a similar estimation procedure may be applied to gamma-Auger coincidence spectra in order to produce an accurate measurement of the Doppler broadening due to the annihilations of positrons with electrons of specific electronic core levels with minimal o-Ps contributions.

\section{Acknowledgments}

The positron beam system at UT Arlington was developed using the NSF major research instrumentation grant DMR-1338130. The research work was funded by NSF grant DMR-1508719. We gratefully acknowledge the support by Welch Foundation (grant No. Y-1968-20180324) for the development of coincidence methods that enabled the experiments described in the manuscript.

\section{References}

[1] T. Hyodo, T. Nakayama, H. Saito, F. Saito, K. Wada, Phys. Status Solidi C 6, 2497 (2009).

[2] V.A. Chirayath, R.W. Gladen, A.D. McDonald, et al., "A multi-stop time-of-flight spectrometer for the measurement of positron annihilation-induced electrons in coincidence with the Doppler broadened annihilation gamma", manuscript under preparation.

[3] T. Hyodo, Mater. Sci. Forum 363, 233 (2001).

[4] S. Xie, Ph.D. Thesis, University of Texas at Arlington, 2002.

[5] R.G. Greaves, C.M. Surko, Can. J. Phys. 74, 445 (1996).

[6] R.W. Gladen, V.A. Chirayath, A.J. Fairchild, A.R. Koymen, A.H. Weiss, Nucl. Instrum. Methods Phys. Res. A 953, 162887 (2020).

[7] P.J. Schultz, K.G. Lynn, Rev. Mod. Phys. 60, 701 (1988). 